\title{
Sonographic Appearance of Extensive Hepatic Arterial Calcification Mimicking Pneumobilia
}

\author{
Sucheta S. Pai, MB, BS, Ronald O. Bude, MD \\ Department of Radiology, University of Michigan Medical Center, 1500 East Medical Center Drive, Ann Arbor, \\ Michigan 48109
}

Received 22 November 2000; accepted 24 July 2001

\begin{abstract}
Pneumobilia appears sonographically as intrahepatic linear echogenic regions that often produce distal acoustic shadowing. We present a case in which extensive hepatic arterial calcification mimicked the sonographic appearance of pneumobilia. In the appropriate clinical setting, extensive hepatic arterial calcification should be considered when intrahepatic linear echogenic regions are found sonographically. (c) 2002 John Wiley \& Sons, Inc. J Clin Ultrasound 30:38-41, 2002.
\end{abstract}

Keywords: liver, calcification; hepatic arteries; ultrasonography

onography is often the preferred modality for $\checkmark$ an initial evaluation of the right upper quadrant of the abdomen, particularly of the biliary system. At times, linear echogenic regions that may branch, may produce distal acoustic shadowing, and occur more centrally than peripherally within the liver are identified; this appearance is most characteristic of pneumobilia. We report a case in which these sonographic findings were produced not by pneumobilia but by extensive hepatic arterial calcification.

\section{CASE REPORT}

A 58-year-old woman was admitted with fever and right upper quadrant pain and tenderness. Her past medical history included myocardial infarction, coronary artery disease, hypertension, hypercholesterolemia, peripheral vascular occlu-

Correspondence to: R. Bude

() 2002 John Wiley \& Sons, Inc.

DOI $10.1002 /$ jcu.10039 sive disease, hyperparathyroidism, and hemodialysis-dependent end-stage renal disease secondary to diabetes requiring hemodialysis for the last 25 years. Abdominal sonography was performed with an HDI 5000 ultrasound scanner (Advanced Technology Laboratories, Bothell, WA) and a $2-5-\mathrm{MHz}$ curved-array transducer. The sonograms revealed linear echogenic foci, many of which were branched, scattered throughout the hepatic parenchyma but with a central predominance (Figure 1). Some of these foci exhibited distal acoustic shadowing. An initial diagnosis of pneumobilia was made.

However, a CT scan performed 1 day later (Figure 2) showed extensive hepatic arterial calcification without evidence of biliary ductal or portal venous gas. Abdominal radiography (Figure 3), performed on the same day as sonography, also demonstrated the extensive calcification in the hepatic artery and the visceral branches of the aorta (the aorta itself had only mild calcification), presumably due to the patient's longstanding renal failure. Subsequent evaluations (hepato-biliary nuclear medicine scan, bilateral lower extremity Doppler sonography, multiple blood cultures, and chest radiography) during her 2-week hospitalization failed to find a cause for the right upper quadrant pain, which subsided after several days. The results of blood cultures showed that she had staphylococcal bacteremia stemming from her dialysis line; this was not considered to be the cause of the pain.

\section{DISCUSSION}

Many conditions produce hepatic calcifications, which often appear sonographically as echogenic regions with or without distal acoustic shadow- 


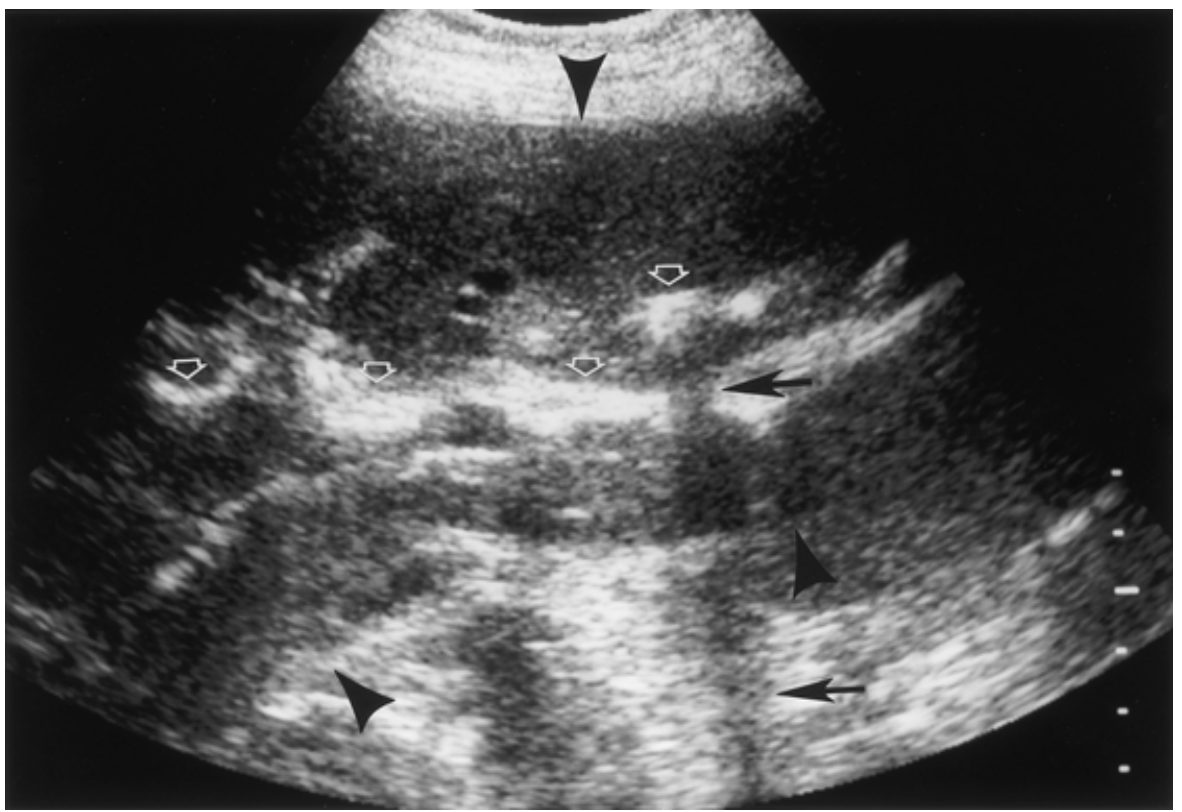

FIGURE 1. Transverse sonogram of the left lobe of the liver (margins denoted with black arrowheads). Open (white) arrows denote the sonographically visualized hepatic arterial calcifications; black arrows denote distal acoustic shadowing arising from 1 of the calcifications.

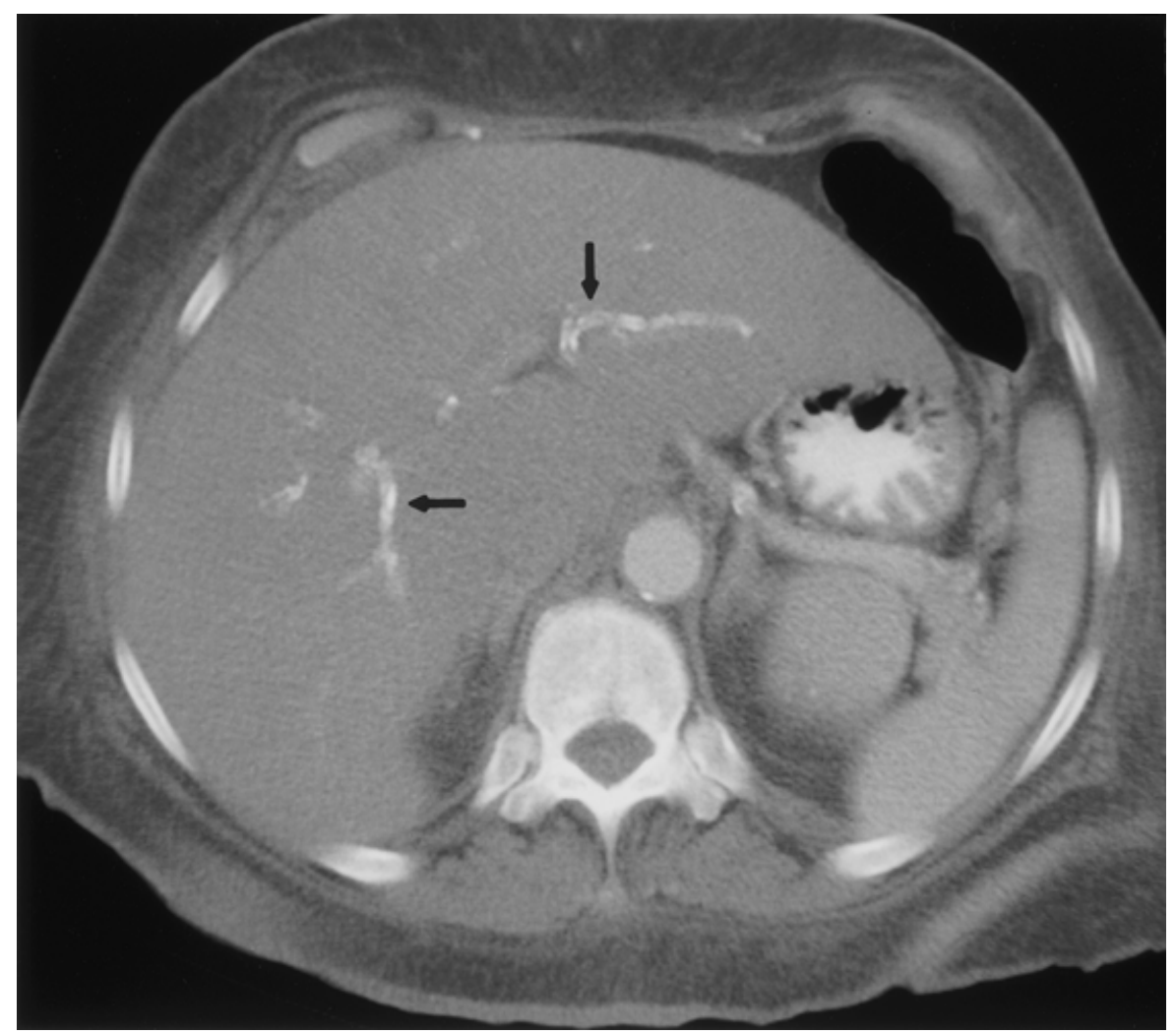

FIGURE 2. Representative CT scan of the liver. Arrows indicate diffuse hepatic arterial calcifications. This image was photographed at wider window settings than were used for scan interpretation, to accentuate the peripheral arterial calcification. Arteries were calcified to this degree throughout the liver.

ing. The causes of such calcifications include echinococci, Schistosoma japonica, calculi within the intrahepatic biliary tree, and tumors such as hepatoblastoma, metastatic colon carcinoma, cystadenocarcinoma, and metastases from VIPomas. $^{1-9}$ The appearance of calcifications in 


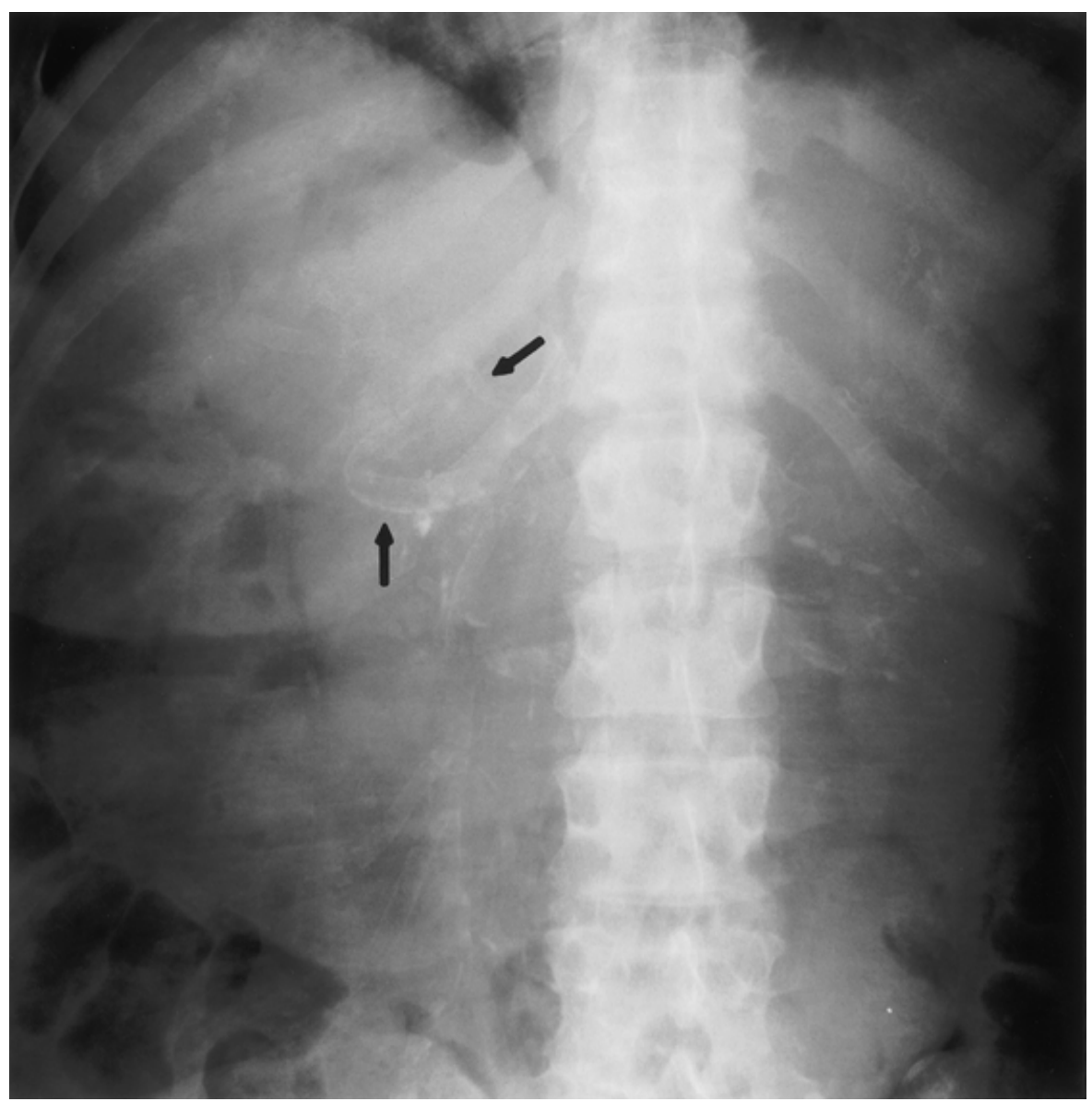

FIGURE 3. Abdominal radiograph. Arrows indicate diffuse arterial calcification throughout branches of the celiac and mesenteric axes, including branches of the hepatic artery.

these usually focal lesions is rarely confused with the sonographic appearance of intrahepatic gas, which usually presents as multiple linear echogenic regions that may branch and that often produce distal acoustic shadowing. Gas in the portal vein produces echogenic foci within the liver, but these foci are usually more peripherally distributed than gas in the biliary system is.

In our patient, sonograms showed multiple linear echogenic regions, many of which produced distal acoustic shadowing, that were predominantly centrally located within the liver. A diagnosis of pneumobilia had been made on the basis of the sonograms, but the true cause of the sonographic abnormality was not appreciated until a CT scan confirmed the absence of pneumobilia and revealed the extensive intrahepatic arterial calcifications.

Two other case reports have described sonographically demonstrable hepatic arterial calcifications. In one ${ }^{10}$ the intrahepatic echogenic foci were more punctate than they were in our case and mimicked intrahepatic biliary calculi. In the other report, ${ }^{11}$ the appearance was not as prominent as it was in our case.

We do not mean to imply that extensive arterial calcification should be suspected whenever multiple linear echogenic regions are seen within the liver on sonograms. This abnormality is almost always produced by pneumobilia. However, if this sonographic abnormality is seen in patients with a clinical history of medical conditions that predispose them to extensive arterial calcification, intrahepatic arterial calcifications should at least be considered during the process of making a differential diagnosis.

\section{REFERENCES}

1. Rizzo AJ, Haller JO, Mulvihill DM, et al. Calcification of the ductus venosus: a cause of right upper quadrant calcification in the newborn. Radiology 1989;173:89.

2. Dachman AH, Pakter RL, Ros PR, et al. Hepatoblastoma: radiologic-pathologic correlation in 50 cases. Radiology 1987;164:15. 
3. Nguyen DL, Leonard JC. Ischemic hepatic necrosis: a cause of fetal liver calcification. AJR Am J Roentgenol 1986;147:596.

4. Albertyn LE, Ganguly BS, Cooper JE, et al. Turtleback liver on CT and ultrasound: Schistosoma japonica revisited. Australas Radiol 1990;34:78.

5. Sasaki F, Ohkawa Y, Sato N, et al. Imaging diagnosis of alveolar echinococcosis in young patients. Pediatr Radiol 1997;27:63.

6. Fassbender CM, Büchsel R, Seelis R, et al. Liver calcifications in metastasizing vipoma. Dtsch Med Wochenschr 1989;114:1445.

7. Naoi Y, Tomita T, Tanaka T, et al. A case report of cystadenocarcinoma of the liver. Gan No Rinsho 1985;31:1332.
8. Schulze K, Hubener KH, Klott K, et al. Computer tomographic and sonographic diagnosis of echinococcus. ROFO: Fortschr Geb Rontgenstr Nuklearmed 1980;132:514.

9. Claudon M, Chaulieu C, Delgoffe C, et al. Role of echography in the diagnosis and monitoring of hepatic alveolar echinococcosis. J Radiol 1984;65:773.

10. White LM, Wilson SR. Hepatic arterial calcification: a potential pitfall in the sonographic diagnosis of intrahepatic biliary calculi. J Ultrasound Med 1994;13:141.

11. Desai RK, Paushter DM, Armistead J. Intrahepatic arterial calcification mimicking pneumobilia: a potential pitfall in the ultrasound evaluation of biliary tract disease. J Ultrasound Med 1989;8:333. 